\title{
Effect of Low-Sodium, Choline-Based Semen Diluent on Viability of Bovine Spermatozoa Stored at $4^{\circ} \mathrm{C}$
}

\author{
Pedro A. Delgado1, Toby D. Lester ${ }^{2}$, Rick W. Rorie ${ }^{2 *}$ \\ ${ }^{1}$ Universidad Publica de El Alto, El Alto, Bolivia \\ ${ }^{2}$ Department of Animal Sciences, University of Arkansas, Fayetteville, AR, USA \\ Email: *rorie@uark.edu
}

How to cite this paper: Delgado, P.A., Lester, T.D. and Rorie, R.W. (2018) Effect of Low-Sodium, Choline-Based Semen Diluent on Viability of Bovine Spermatozoa Stored at $4^{\circ} \mathrm{C}$. Advances in Reproductive Sciences, 6, 12-21.

http://dx.doi.org/10.4236/arsci.2018.61002

Received: December 20, 2017

Accepted: February 24, 2018

Published: February 27, 2018

Copyright $\odot 2018$ by authors and Scientific Research Publishing Inc. This work is licensed under the Creative Commons Attribution International License (CC BY 4.0).

http://creativecommons.org/licenses/by/4.0/

(c) (i) Open Access

\begin{abstract}
This study evaluated sperm viability over time, after dilution and refrigerator storage of fresh semen extended in either synthetic cauda epididymal plasma (CEP2), or in a low sodium medium (CJ2) supplemented with either AlbuMAX or egg yolk. Semen collected weekly for 4 weeks from 4 bulls and assigned within bulls, across treatments. After extension in either CEP2, or CJ2 containing either egg yolk or AlbuMAX, semen was cooled to $4^{\circ} \mathrm{C}$, and evaluated for 7 days. A computer assisted sperm analysis (CASA) system was used for sperm evaluation. Particular emphasis was placed on sperm motility since it is the single most important sperm parameter influencing bull fertility. Total and progressive motility of sperm in CEP2 and CJ2-AlbuMAX were similar ( $\mathrm{P}=0.85$ and $\mathrm{P}=0.23$, respectively), but both were lower $(\mathrm{P}<0.01)$ when compared to CJ2-yolk. Fewer sperm had rapid motility in CEP2 and CJ2-AlbuMAX compared to CJ2-yolk $(\mathrm{P}<0.01)$. Sperm straightness and linearity were greater in CJ2-AlbuMAX and CJ2-yolk than in CEP2 $(\mathrm{P}<0.01)$. Mean velocity (VAP) and linear velocity (VSL) were greater $(\mathrm{P}<0.01)$ in CJ2-AlbuMAX than either CEP2 or CJ2-yolk. The calculated curvilinear velocity (VCL) of spermatozoa in CEP2 was lower than CJ2-AlbuMAX (P = $0.01)$, but similar with $\mathrm{CJ} 2$-yolk $(\mathrm{P}=0.54)$. Overall, every sperm parameter measured by the CASA system was equal to or higher for sperm stored 7 days in CJ2 medium as compared with CEP2. The CJ2 extender supplemented with egg yolk is a viable alternative for storing fresh bovine semen.
\end{abstract}

\section{Keywords}

Semen Extender, Bovine Sperm, Refrigerator Storage 


\section{Introduction}

Approximately $50 \%$ of viable spermatozoa are detrimentally affected after exposure to either fresh or frozen storage procedures [1] [2]. Semen handling and extender composition are crucial factors that affect spermatozoa after dilution [3] [4]. Studies had been conducted to define an optimal extender for storing fresh bull semen, and several have reported on extenders that improve sperm survival during prolonged storage [4] [5] [6] [7]. However, few of these extenders prevented damage to the spermatozoa after dilution, resulting in eventual reduction in sperm quality parameters such as total and progressive motility after 5 or $6 \mathrm{~d}$ of storage [6] [8].

Ionic compounds present in the storage medium can cause cell damage during cooling or freezing procedures. Some cryopreservation studies have reported that sodium ions can cause adverse effects during cooling and re-warming of oocytes or embryos [9] [10] [11] [12]. Sodium ions can cross through specialized ion channels in the cell membrane and become toxic at high concentration inside the cell [13]. Replacing sodium ions with choline chloride in a medium improves viability of animal cells subjected to cooling or freezing procedures [9]. Choline chloride is an organic compound similar to sodium that possesses protective properties against cold exposure, but apparently cannot cross cell membranes [14]. Sodium ions are also common compounds in sperm preservation media, and possibly have the same adverse effects on sperm as those reported for other cell types. Thus, it might be beneficial to replace sodium chloride with choline chloride in sperm preservation medium.

Another ingredient extensively used for sperm protection during cooling and freezing procedures is egg yolk. Several studies have reported the benefits of using egg yolk in semen extenders [1] [15] [16]. Egg yolk has low-density lipoproteins that help stabilize the plasma membrane of sperm [17], but also bind toxic components from the seminal plasma and extender [5] [15] [18]. Because egg yolk can be a source of bacterial contamination in semen extenders, some researchers have looked for substitutes such as soy lecithin. Another possible substitute for egg yolk could be the lipid-rich bovine serum albumin known as AlbuMAX. Lim et al. [12] found AlbuMAX to be superior to either serum albumin or serum in protecting embryos during cooling and freezing procedures. They reported a significant improvement in embryo survival and higher transfer pregnancy rates after cryopreservation in media based on choline chloride and supplemented with AlbuMAX.

We hypothesize that the concentration of sodium ions in current extenders are harmful to the function and viability of spermatozoa. Replacing sodium content in media with choline chloride and replacing egg yolk with AlbuMAX in the extender might help to maintain viability of sperm for extended periods of time. Therefore, this study evaluated sperm viability over time, after dilution and refrigerator storage $\left(4^{\circ} \mathrm{C}\right)$ of fresh semen extended in either synthetic cauda epididymal plasma (CEP2) or in a low sodium medium (CJ2) supplemented with 
either AlbuMAX or egg yolk.

\section{Materials and Methods}

\subsection{Semen Collection and Processing}

Semen was collected by electroejaculation, from 6 mature Angus bulls. After evaluation of semen samples with computer assisted sperm analysis (CASA), 4 bulls, ranging in age from 3 to 6 years, weighing between 632 and $884 \mathrm{~kg}$, and in moderate to good body condition were selected for use in the study that represented a range in semen quality. During the experiment, semen was collected each week for 4 weeks. Among the bulls, the percent motile and progressive sperm ranged from $53 \%$ to $81 \%$, and $34 \%$ to $69 \%$, respectively. All semen samples were initially evaluated within $1 \mathrm{~h}$ of collection. The semen samples were not washed of seminal plasma, but were directly extended in each treatment.

\subsection{Semen Extenders and Treatments}

The experimental treatments were fresh semen preservation at $4^{\circ} \mathrm{C}$ in synthetic cauda epididymal plasma (CEP2) [19], or in essentially sodium-free CJ2 medium [9] supplemented with either egg yolk or AlbuMAX. Formulations for these media are presented in Table 1. All media ingredients were purchased from Sigma Chemical (St. Louis, MO, USA). Both media were prepared as 2x stock solutions without the energy sources, gentamicin, AlbuMAX or egg yolk. After addition of these ingredients to $50 \mathrm{ml}$ of the appropriate media, the volume was brought to $100 \mathrm{ml}$ with Milli-Q water, the $\mathrm{pH}$ was adjusted to 6.6, and the media was filter sterilized. In media containing egg yolk, the volume was brought to $90 \mathrm{ml}$, the $\mathrm{pH}$ adjusted and filter sterilized before addition of egg yolk. Media were prepared fresh weekly throughout the study and stored in a refrigerator $\left(4^{\circ} \mathrm{C}\right)$ until use. After the initial evaluation, an aliquot of semen containing 60 million spermatozoa $\left(20 \times 10^{6} \mathrm{sperm} / \mathrm{ml}\right)$ from each bull was assigned across treatments. The semen and extender treatments (CEP2, CJ2-AlbuMAX, and CJ2-yolk) were equilibrated to $35^{\circ} \mathrm{C}$ before mixing in $5 \mathrm{ml}$ polystyrene snap-top culture tubes. The sealed tubes were then placed into a beaker containing $300 \mathrm{ml}$ of water at $35^{\circ} \mathrm{C}$, and placed into a refrigerator at $4^{\circ} \mathrm{C}$ to cool slowly over $3 \mathrm{~h}$. During storage, the stored samples were covered to prevent potential damage due to light.

\subsection{Evaluation of Sperm Quality Parameters}

An aliquot of $50 \mu \mathrm{l}$ of semen was removed from each extender treatment on days $1,3,4,5$ and 7 of refrigerated storage to measure sperm parameters. A Hamilton Thorne Biosciences IVOS computer-assisted sperm analysis (CASA) system with version 12 TOX IVOS software was used. The parameters measured were motility (\%), progressive motility (\%), velocity distribution (rapid \%), path velocity (VAP $\mu \mathrm{m} / \mathrm{s}$ ), velocity straight line (VSL $\mu \mathrm{m} / \mathrm{s}$ ), track speed (VCL $\mu \mathrm{m} / \mathrm{s}$ ), lateral amplitude (ALH $\mu \mathrm{m})$, beat cross frequency (BCF Hz), straightness (STR \%), and 
Table 1. Composition of CEP2 and CJ2 semen extenders.

\begin{tabular}{|c|c|c|c|}
\hline Component & CEP2 & CJ2-AlbuMAX & CJ2-yolk \\
\hline Trisma (TRIS Base), $\mathrm{mM}$ & 133.70 & 0.0 & 0.0 \\
\hline C5H14NOCl (Choline Chloride), $\mathrm{mM}$ & 0.0 & 137.93 & 137.93 \\
\hline $\mathrm{NaCl}, \mathrm{mM}$ & 15.00 & 0.0 & 0.0 \\
\hline $\mathrm{KCl}, \mathrm{mM}$ & 7.00 & 2.67 & 2.67 \\
\hline $\mathrm{CaCl}_{2}{ }^{\star} 2 \mathrm{H}_{2} \mathrm{O}, \mathrm{mM}$ & 3.00 & 0.0 & 0.0 \\
\hline $\mathrm{MgCl}_{2}{ }^{\star} 6 \mathrm{H}_{2} \mathrm{O} \mathrm{mM}$ & 4.00 & 0.495 & 0.495 \\
\hline $\mathrm{NaHCO}_{3}, \mathrm{mM}$ & 11.90 & 0.0 & 0.0 \\
\hline $\mathrm{NaH}_{2} \mathrm{PO}_{4}, \mathrm{mM}$ & 8.00 & 0.0 & 0.0 \\
\hline $\mathrm{KH}_{2} \mathrm{PO}_{4}, \mathrm{mM}$ & 20.00 & 1.47 & 1.47 \\
\hline $\mathrm{Na}_{2} \mathrm{HPO}_{4}, \mathrm{mM}$ & 0.0 & 8.06 & 8.06 \\
\hline Fructose, $\mathrm{mM}$ & 55.00 & 55.00 & 55.00 \\
\hline Ca lactate, $\mathrm{mM}$ & 0.0 & 1.00 & 1.00 \\
\hline Sorbitol (g/l) & 1.00 & 1.00 & 1.00 \\
\hline BSA (\%) & 0.20 & 0.0 & 0.0 \\
\hline Na pyruvate & 0.0 & 1.00 & 1.00 \\
\hline AlbuMAX (\%) & 0.0 & 1.00 & 0.0 \\
\hline Citric Acid, mM & 42.90 & 0.0 & 0.0 \\
\hline Gentamicin $(10 \mathrm{mg} / \mathrm{ml} ; \mathrm{ml} / \mathrm{l})$ & 5.00 & 5.00 & 5.00 \\
\hline Fresh egg yolk (\% v/v) & 10.00 & 0.0 & 10.00 \\
\hline
\end{tabular}

linearity (LIN \%). In order to accurately measure sperm parameters in the presence of egg yolk (some egg yolk globules may be identified as immobile spermatozoa) extended semen samples were stained with Hoechst 33342 before analysis (IVOS IDENT illumination option). A $50 \mu \mathrm{l}$ aliquot of semen from each treatment were warmed for 10 minutes at $35^{\circ} \mathrm{C}$, mixed with $50 \mu \mathrm{l}$ of pre-warmed Dulbeco's PBS, and then mixed with $1 \mu \mathrm{l}$ of a $10 \mu \mathrm{g} / \mathrm{ml}$ solution of Hoechst 33342. After a 10 min equilibration with the Hoechst stain at $35^{\circ} \mathrm{C}$, the sample was analyzed using the CASA system. For analysis, 8 fields were scanned, with 30 video frames captured per field. A minimum of 400 spermatozoa was counted in each sample.

\subsection{Statistical Analysis}

Data from the CASA sperm quality parameters were analyzed using GLM procedure of Statistical Analysis System software [20]. A linear mixed effect model was used. Independent factors were the extender treatments (CJ2-AlbuMAX, CJ2-yolk and CEP2) and days of evaluation (1, 3, 4, 5, and $7 \mathrm{~d}$ of refrigerated storage). The replications (bulls) were blocked to control variation and tested as experimental error in the model. Dependent variables $\left(Y_{i j}\right)$ measured with IVOS 
were: motility (\%), progressive motility (\%), velocity distribution (rapid \%), path velocity (VAP $\mu \mathrm{m} / \mathrm{s}$ ), velocity straight line (VSL $\mu \mathrm{m} / \mathrm{s}$ ), track speed (VCL $\mu \mathrm{m} / \mathrm{s}$ ), lateral amplitude $(\mathrm{ALH} \mu \mathrm{m})$, beat cross frequency (BCF $\mathrm{Hz}$ ), straightness (STR \%), and linearity (LIN \%). Subjective observation for abnormalities, live and dead sperm also were analyzed as dependent variables. Variables that did not meet statistical assumptions were transformed to logarithmic scale ( $\log 10$ $(\mathrm{X}+1)$ ) and angular scale (Arcsine(SQRT $(\mathrm{X} / 100)$. Differences among extenders and days were tested by LSmeans analysis procedure at 0.05 of $\alpha$. Original and transformed data were analyzed using the following model:

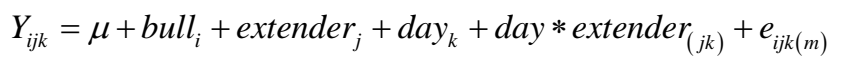

\section{Results}

There were no interactions $(\mathrm{P}>0.05)$ between days and extenders on sperm variables expressed as percent. For total and rapid motility, no differences $(\mathrm{P}>$ $0.05)$ were observed among bulls. However, progressive, straight, and linear motility were different among bulls $(\mathrm{P}<0.05)$. Therefore, the main effects of extenders on sperm variables during $7 \mathrm{~d}$ of fresh storage were compared (Table 2). Total and progressive motility of spermatozoa in CEP2 and CJ2-AlbuMAX extenders were similar ( $\mathrm{P}=0.85$ and $\mathrm{P}=0.23$ respectively), but both were significantly lower $(\mathrm{P}<0.01)$ compared to $\mathrm{CJ} 2$-yolk. The percentage of spermatozoa with rapid motility was lower $(\mathrm{P}<0.01)$ in CEP2 and CJ2-AlbuMAX extenders compared to CJ2-yolk. Spermatozoa straightness and linearity were similar ( $\mathrm{P}=$ 0.48 and $\mathrm{P}=0.06$ respectively) in CJ2-AlbuMAX and in CJ2-yolk extenders; however, spermatozoa in CEP2 presented less $(\mathrm{P}<0.01)$ straightness and linearity compared with spermatozoa of the other treatments.

No interactions between days and extender treatments occurred for sperm VAP, VSL, VCL, ALH, and BCF parameters $(\mathrm{P}>0.05)$, and there was no difference of VAP, VCL, and ALH among bulls (P > 0.05). Only VSL, and BCF showed statistical differences $(\mathrm{P}<0.05)$. Therefore, the main effect of extender treatments on these parameters during $7 \mathrm{~d}$ of fresh storage was compared (Table 3 ). The mean path velocity (VAP) of spermatozoa was similar in extender CEP2 and CJ2-yolk $(\mathrm{P}=0.33)$, but spermatozoa VAP was greater $(\mathrm{P}<0.01)$ than either in CJ2-AlbuMAX. The calculated linear (straight line) velocity (VSL) of spermatozoa in CJ-2-AlbuMAX was superior $(\mathrm{P}<0.01)$ to CJ2-yolk and CEP2 treatments. Spermatozoa in CJ2-yolk also had a higher VSL value than those in the CEP2 treatment $(P=0.01)$. The calculated curvilinear (track speed) velocity (VCL) of spermatozoa in CEP2 was lower than CJ2-AlbuMAX (P = 0.01), but it was similar with CJ2-yolk $(\mathrm{P}=0.54)$. Spermatozoa in CJ2-AlbuMAX and CJ2-yolk also showed similar VCL $(\mathrm{P}=0.06)$. The mean width of lateral sperm head oscillation (ALH) in CEP2 was statistically similar to CJ2-AlbuMAX and CJ2-yolk ( $\mathrm{P}=0.95$ and $\mathrm{P}=0.69$ respectively). The number of lateral oscillatory movements of the sperm head around the mean trajectory (beat cross frequency; BCF) was higher in CJ2-yolk extender compared with CJ2-AlbuMAX and CEP2 
Table 2. Overall mean percentages for sperm parameters of bull semen preserved in CEP2, CJ2-AlbuMAX, or CJ2-yolk extenders after 7 days of fresh preservation.

\begin{tabular}{cccc}
\hline & \multicolumn{3}{c}{ Extenders } \\
\hline Sperm characteristics & CEP2 & CJ2-AlbuMAX & CJ2-yolk \\
Total motility \% & $32.57 \pm 1.74^{\mathrm{b}}$ & $32.85 \pm 1.66^{\mathrm{b}}$ & $41.16 \pm 1.72^{\mathrm{a}}$ \\
Progressive motility \% & $21.72 \pm 1.48^{\mathrm{b}}$ & $23.44 \pm 1.43^{\mathrm{b}}$ & $29.05 \pm 1.47^{\mathrm{a}}$ \\
Rapid motility \% & $27.96 \pm 1.69^{\mathrm{b}}$ & $28.89 \pm 1.63^{\mathrm{b}}$ & $34.56 \pm 1.68^{\mathrm{a}}$ \\
Straightness \% & $78.19 \pm 0.78^{\mathrm{b}}$ & $83.70 \pm 0.75^{\mathrm{a}}$ & $84.38 \pm 0.77^{\mathrm{a}}$ \\
Linearity \% & $46.63 \pm 1.00^{\mathrm{b}}$ & $53.70 \pm 0.97^{\mathrm{a}}$ & $51.04 \pm 0.99^{\mathrm{a}}$ \\
\hline
\end{tabular}

${ }^{\mathrm{a}, \mathrm{b}}$ Each value given is the mean \pm S.E.M. Values with different alphabetical superscripts within rows indicate a statistical difference.

Table 3. Overall mean percentages for sperm parameters of bull semen preserved in CEP2, CJ2-AlbuMAX, or CJ2-yolk extenders after $7 \mathrm{~d}$ of fresh preservation.

\begin{tabular}{cccc}
\hline & \multicolumn{3}{c}{ Extenders } \\
\hline Sperm characteristics & CEP2 & CJ2-AlbuMAX & CJ2-yolk \\
Path velocity (VAP, $\mu \mathrm{m} / \mathrm{s})$ & $86.11 \pm 2.22^{\mathrm{b}}$ & $101.12 \pm 2.13^{\mathrm{a}}$ & $87.86 \pm 2.19^{\mathrm{b}}$ \\
Straight line velocity $(\mathrm{VSL}, \mu \mathrm{m} / \mathrm{s})$ & $68.66 \pm 2.14^{\mathrm{c}}$ & $85.97 \pm 2.06^{\mathrm{a}}$ & $74.15 \pm 2.12^{\mathrm{b}}$ \\
Track speed $(\mathrm{VCL}, \mu \mathrm{m} / \mathrm{s})$ & $152.19 \pm 3.81^{\mathrm{b}}$ & $165.33 \pm 3.66^{\mathrm{a}}$ & $153.86 \pm 3.77^{\mathrm{ab}}$ \\
Lateral amplitude $(\mathrm{ALH}, \mu \mathrm{m})$ & $6.55 \pm 0.16^{\mathrm{a}}$ & $6.49 \pm 0.16^{\mathrm{a}}$ & $6.36 \pm 0.16^{\mathrm{a}}$ \\
Beat cross frequency $(\mathrm{BCF}, \mathrm{Hz})$ & $20.74 \pm 0.96^{\mathrm{c}}$ & $24.40 \pm 0.92^{\mathrm{b}}$ & $30.36 \pm 0.95^{\mathrm{a}}$ \\
\hline
\end{tabular}

a,b,cEach value given is the mean \pm S.E.M. Values with different alphabetical superscripts within rows indicate a statistical difference between treatments $(\mathrm{P}<0.05)$.

extenders $(\mathrm{P}<0.01)$. Spermatozoa in CJ2-AlbuMAX had higher BCF than in $\mathrm{CEP} 2(\mathrm{P}<0.01)$.

\section{Discussion}

About $95 \%$ of the bovine semen used in the developed world is cryopreserved, with the remaining $5 \%$ used as fresh semen [21]. In order to achieve an acceptable pregnancy rate and compensate for damage caused during the freezing and thawing process, an insemination dose of $\sim 15$ million spermatozoa are needed when using frozen semen. An advantage of using fresh semen is that an insemination dose of 2 to 4 million spermatozoa is adequate for acceptable pregnancy rates. Although fresh semen can be stored for up to 3 days at room temperature (under nitrogen gas) in commercially available Caprogen extender [1], most prefer storage at refrigerator temperatures to extend shelf life.

The bovine cauda epididymis has the ability to store semen for several weeks without dramatic reduction in viability [22]. Synthetic cauda epididymal plasma (CEP) is a fresh semen extender that was developed based on analysis of epididymal fluid [23]. A variation of this fresh semen extender (CEP2) was shown to be superior in maintaining sperm motility and membrane integrity as compared 
to a commonly used Tris-based extender [2], so it was chosen for comparison in the present study.

The other medium used in this study (CJ2) was developed, based on the finding that sodium ions damage cellular integrity and developmental competence of cryopreserved mouse oocytes [9]. Sodium ions can cross through ion channels in the cell membrane and become toxic at high concentration inside the cell. Stachecki et al. [9] created CJ2 by taking the formulation of Dulbecco's PBS and replacing sodium chloride with choline chloride, and by replacing the mono and dibasic sodium phosphate buffers with potassium phosphate to create a sodium-free medium. Choline chloride is an organic compound similar to sodium that possesses protective properties against cold exposure, but apparently cannot cross cell membranes [12] [14]. Sodium ions are common in sperm preservation media, and may also have adverse effects on spermatozoa. Therefore, CJ2 was chosen for evaluation as an extender for fresh semen.

Most semen extenders used for either fresh or cryopreserved semen contain varying percentages of fresh egg yolk. Egg yolk contains lipoproteins that stabilize the plasma membrane of sperm [17], bind toxic components from the seminal plasma and extender [15] and help prevent hypermotility and capacitation [24] [25]. Unfortunately, egg yolk can also be a source of bacterial contamination in semen extenders; some researchers have looked for substitutes. AlbumMAX is a lipid rich form of bovine serum albumin containing $0.65 \%$ lipids by weight, including free fatty acids, lysophosphatidylcholine, triacylglycerides, phosphatidylcholine, phosphatidic acid, and cholesterol [26]. Due to its lipid content, AlbuMAX was evaluated as a possible substitute for egg yolk in this study.

Comparison of total, progressive and rapid motility over 7 days of storage indicated that the low-sodium CJ2 medium supplemented with egg was superior to CEP2 extender. Motility is the single most important parameter in predicting fertility [27]. The CJ2 supplemented with 1\% AlbuMAX was equal to CEP2 in maintaining motility of stored spermatozoa. These results suggest that CJ2 is a viable alternative as an extender for fresh semen. Egg yolk was used in the CEP2 and CJ2-yolk at $10 \%$ of total volume, whereas AlbuMAX was used at $1 \%$. Further study is needed to determine whether higher concentrations of AlbuMAX would be beneficial in maintaining sperm motility during storage. Both sperm path velocity (VAP) and straight line velocity (VSL) were greater in CJ2-AlbuMAX, while the other two treatments supplemented with egg yolk were similar. The other velocity measurement, speed over the actual sperm track (VCL) was similar for both CJ2 treatments, with both greater than the CEP treatment. The differences noted in VAP and VCL might be explained by the absence of egg yolk in the CJ2-AlbuMAX extender. As mentioned above, egg yolk is known to help prevent hyper motility [24] [25]. Therefore, it might be expected that spermatozoa stored in extenders containing egg yolk would have lower velocity. A function of extenders is to suppress motility and sperm metabolism 
during sperm storage. It would appear that the CJ2-AlbuMAX extender was not detrimental, since the velocity measures were higher even after 7 days of storage.

\section{Conclusion}

In summary, every sperm parameter measured by the CASA system was equal to or higher for spermatozoa stored 7 days in CJ2 medium as compared to CEP2. Therefore, it can be concluded that CJ2 supplemented with egg yolk is a viable alternative to other semen extenders for storage of fresh semen. Additional studies are needed to determine if a higher concentration of AlbuMAX would be equal to egg yolk in maintaining sperm motility. Also, studies are needed to determine if sperm stored in CJ2 medium would result in acceptable pregnancy rates after artificial insemination.

\section{Acknowledgements}

The authors acknowledge the University of Arkansas Agricultural Experiment Station and the Department of Animal Science for their financial support.

\section{References}

[1] Vishwanath, R. and Shanon, P. (2000) Storage of Bovine Semen in Liquid and Frozen State. Animal Reproduction Science, 62, 23-53. https://doi.org/10.1016/S0378-4320(00)00153-6

[2] Verberckmoes, S., Van Soom, A., Dewulf, J., De Pauw, I.M.C. and De Kruif, A. (2004) Storage of Fresh Bovine Semen in a Diluent Based on the Ionic Composition of Cauda Epididymal Plasma. Reproduction Domestic Animals, 39, 410-416. https://doi.org/10.1111/j.1439-0531.2004.00521.x

[3] Guthrie, H.D., Liu, J. and Crister, J.K. (2002) Osmotic tolerance limits and effects of cryoprotectants on motility of bovine spermatozoa. Biology of Reproduction, 67, 1811-1816. https://doi.org/10.1095/biolreprod67.6.1811

[4] Celeghini, E.C., de Arruda, R.P., de Andrade, A.F., Nascimento, J., Raphael, C.F. and Rodrigues, P.H. (2008) Effects That Bovine Sperm Cryopreservation Using Two Different Extenders Has on Sperm Membranes and Chromatin. Animal Reproduction Science, 104, 119-131. https://doi.org/10.1016/j.anireprosci.2007.02.001

[5] Thun, R., Hurtado, M. and Janett, F. (2002) Comparison of Biociphos-Plus ${ }^{\circledR}$ and TRIS-Egg Yolk Extender for Cryopreservation of Bull Semen. Theriogenology, 57, 1087-1094. https://doi.org/10.1016/S0093-691X(01)00704-X

[6] Verberckmoes, S., Van Soom, A., Dewulf, J. and De Kruif, A. (2005) Comparison of Three Diluents for the Storage of Fresh Bovine Semen. Theriogenology, 63, 912-922. https://doi.org/10.1016/j.theriogenology.2004.05.011

[7] Chaveiro, A., Machado, L., Frijters, A., Engel, B. and Woelders, H. (2006) Improvement of Parameters of Freezing Medium and Freezing Protocol for Bull Sperm Using Two Osmotic Supports. Theriogenology, 65, 1875-1890. https://doi.org/10.1016/j.theriogenology.2005.10.017

[8] Nair, S.J., Brar, A.S., Ahuja, C.S., Sangha, S.P.S. and Chaudhary, K.C. (2006) A Comparative Study on Lipid Peroxidation, Activities of Antioxidant Enzymes and Viability of Cattle and Buffalo Bull Spermatozoa during Storage at Refrigeration Temperature. Animal Reproduction Science, 96, 21-29.

https://doi.org/10.1016/j.anireprosci.2005.11.002 
[9] Stachecki, J.J., Cohen, J. and Willadsen, S.M. (1998) Cryopreservation of Unfertilized Mouse Oocytes: The Effect of Replacing Sodium with Choline in the Freezing Medium. Cryobiology, 37, 346-354. https://doi.org/10.1006/cryo.1998.2130

[10] Stachecki, J.J. and Willadsen, S.M. (2000) Cryopreservation of Mouse Oocytes Using a Medium with Low Sodium Content: Effect of Plunge Temperature. Cryobiology, 40, 4-12. https://doi.org/10.1006/cryo.1999.2215

[11] Stachecki, J.J., Cohen, J. and Munne, S. (2005) Cryopreservation of Biopsied Cleavage Stage Human Embryos. Reproductive Biomedicine Online, 11, 711-715. https://doi.org/10.1016/S1472-6483(10)61689-6

[12] Lim, K.T., Jang, G., Ko, K.H., Lee, W.W., Park, H.J., Kim, J.J., Kang, S.K. and Lee, B.C. (2008) Improved Cryopreservation of Bovine Preimplantation Embryos Cultured in Chemically Defined Medium. Animal Reproduction Science, 103, 239-248. https://doi.org/10.1016/j.anireprosci.2006.12.020

[13] Stachecki, J.J., Cohen, J., Schimmel, T. and Willadsen, S.M. (2002) Fetal Development of Mouse Oocytes and Zygotes Cryopreserved in a Nonconventional Freezing Medium. Cryobiology, 44, 5-13. https://doi.org/10.1016/S0011-2240(02)00007-X

[14] Toner, M., Cravalho, E.G., Stachecki, J., Fitzgerald, T., Tompkins, R.G., Yarmush, M.L. and Armant, D.R. (1993) Nonequilibrium Freezing of One-Cell Mouse Embryos. Biophysiology Journal, 64, 1908-1921. https://doi.org/10.1016/S0006-3495(93)81562-5

[15] Manjunath, P., Nauc, V., Bergeron, A. and Menard, M. (2002) Major Proteins of Bovine Seminal Plasma Bind the Low-Density Lipoprotein Fraction of Hen's Egg Yolk. Biology of Reproduction, 67, 1250-1258.

https://doi.org/10.1095/biolreprod67.4.1250

[16] Bergeron, A., Crete, M.H., Brindle, Y. and Manjunath, P. (2004) Low-Density Lipoprotein Fraction from Hen's Egg Yolk Decreases the Binding of the Major Proteins from Bovine Seminal Plasma to Sperm and Prevents Lipid Efflux from the Sperm Membrane. Biology of Reproduction, 70, 708-717. https://doi.org/10.1095/biolreprod.103.022996

[17] Bencharif, D., Amirat, L., Anton, M., Schmitt, E., Desherces, S., Delhomme, D., Langlois, M.L., Barriere, P., Larrat, M. and Taintuier, D. (2008) The Advantages of LDL (Low Density Lipoproteins) in the Cryopreservation of Canine Semen. Theriogenology, 70, 1478-1488. https://doi.org/10.1016/j.theriogenology.2008.06.095

[18] De Pauw, I.M., Van Soom, A., Mintiens, K., Verberckmoes, S. and de Kruif, A. (2003) In Vitro Survival of Bovine Spermatozoa Stored at Room Temperature under Epididymal Conditions. Theriogenology, 59, 1093-1107. https://doi.org/10.1016/S0093-691X(02)01207-4

[19] Verberckmoes, S., Van Soom, A., Dewulf, J., De Pauw, I. and de Kruif, A. (2004) Storage of Fresh Bovine Semen in a Diluent Based on the Ionic Composition of Cauda Epididymal Plasma. Reproduction Domestic Animals, 39, 410-416. https://doi.org/10.1111/j.1439-0531.2004.00521.x

[20] SAS (2003) User's Guide: Statistics, Version 9.1 Ed. SAS Institute, Inc., Cary, NC.

[21] Chuppin, D. and Thibier, M. (1995) Survey of the Present Status of the Use of Artificial Insemination in Developed Countries. Revista Mundial de Zootecnia (FAO), 82, 58-68. http://www.fao.org/3/a-v4440t/v4440T0m.htm

[22] Kirillov, V.S. and Mozorov, V.A. (1936) Duration of Survival of Bull Spermatozoa in an Epididymis Isolated from the Testis. Animal Breeding, 5, 22 (Abstr.).

[23] Verberckmoes, S., De Pauw, I., Van Soom, A. and De Kruif, A. (2001) Ionic Composition and Osmolarity of Caudal Epididymal Plasma in the Bull. Theriogenology, 55, 449 (Abstr). 
[24] Amirat, L., Tainturier, D., Jeanneau, L., Thorin, Ch., Gerard, O., Courtens, J.L. and Anton, M. (2004) Bull Semen in Vitro Fertility after Cryopreservation Using Egg Yolk LDL a Comparison with Optidyl a Commercial Egg Yolk Extender. Theriogenology, 61, 895-907. https://doi.org/10.1016/S0093-691X(03)00259-0

[25] Ho, H.C., Granish K.A. and Suarez, S.S. (2002) Hyperactivated Motility of Bull Sperm Is Triggered at the Axoneme by $\mathrm{Ca}^{2+}$ and Not cAMP. Developmental Biolo$g y, 250,208-217$. https://doi.org/10.1006/dbio.2002.0797

[26] Garcia-Gonzalo, F.R. and Izpisua-Belmonte, J.C. (2008) Albumin-Associated Lipids Regulate Human Embryonic Stem Cell Self-Renewal. PLoS ONE, 3, e1384. https://doi.org/10.1371/journal.pone.0001384

[27] Farrell, P.B., Presicce, G.A., Brockett, C.C. and Foote, R.H. (1998) Quantification of Bull Sperm Characteristics Measured by Computer-Assisted Sperm Analysis (CASA) and the Relationship to Fertility. Theriogenology, 49, 871-879.

https://doi.org/10.1016/S0093-691X(98)00036-3 